SUBJECT AREAS:

SPINTRONICS

ELECTRONIC DEVICES

Received

11 March 2014

Accepted

16 June 2014

Published

7 July 2014

Correspondence and requests for materials should be addressed to P.S.A.K. (anil@ physics.iisc.ernet.in) \title{
injection into GaAs by an oxide spin injector
}

\author{
Shwetha G. Bhat \& P. S. Anil Kumar
}

Department of Physics, Indian Institute of Science, Bangalore-560012, INDIA.

Spin injection, manipulation and detection are the integral parts of spintronics devices and have attracted tremendous attention in the last decade. It is necessary to judiciously choose the right combination of materials to have compatibility with the existing semiconductor technology. Conventional metallic magnets were the first choice for injecting spins into semiconductors in the past. Here we demonstrate the electrical spin injection from an oxide magnetic material $\mathrm{Fe}_{3} \mathrm{O}_{4}$, into $\mathrm{GaAs}$ with the help of tunnel barrier $\mathrm{MgO}$ at room temperature using 3-terminal Hanle measurement technique. A spin relaxation time $\tau \sim 0.9 \mathrm{~ns}$ for $\mathrm{n}$-GaAs at $300 \mathrm{~K}$ is observed along with expected temperature dependence of $\tau$. Spin injection using $\mathrm{Fe}_{3} \mathrm{O}_{4} /$ $\mathrm{MgO}$ system is further established by injecting spins into $\mathrm{p}-\mathrm{GaAs}$ and a $\tau$ of $\sim 0.32 \mathrm{~ns}$ is obtained at $300 \mathrm{~K}$. Enhancement of spin injection efficiency is seen with barrier thickness. In the field of spin injection and detection, our work using an oxide magnetic material establishes a good platform for the development of room temperature oxide based spintronics devices.

E ver since the proposal of spin field effect transistor by Datta and Das in the year $1990^{1}$, the research on spin injection and detection has been one of the central themes in condensed matter physics and materials science $^{2-6}$. Initially, optical methods of spin injection and detection in semiconductors ${ }^{7,8}$ were studied extensively by several groups; but, later on as the research progressed in this field, electrical injection methods were attempted and became successful ${ }^{9-11}$ though detection techniques still remained as optical. The non-local electrical measurements on metallic spin-valves by Jedema F. J. et al ${ }^{12}$ in 2002 demonstrated that both spin injection and detection can be carried out completely by electrical methods, which proved to be more efficient because of the ease of integration and practical usage of these devices.

However, in case of spin injection into semiconductors, lack of sufficient spin injection efficiency due to the conductivity mismatch between the metallic magnets and the semiconductors were the initial hurdles encountered by the research community. Hence, overcoming the conductivity mismatch ${ }^{13,14}$ between the spin injector and the semiconductor or using an injection material with high spin polarization would be the efficient ways of injecting spins into semiconductors. It is now established that one can inject spins into conventional semiconductors such as $\mathrm{Si}, \mathrm{Ge}$, and $\mathrm{GaAs}$ etc. using ferromagnetic electrodes such as $\mathrm{CoFeB}, \mathrm{Co}, \mathrm{NiFe}$ etc. through a tunnel barrier ${ }^{4-6}$. Recently, J. Bae et al..$^{15}$ also observed electrical spin injection into GaAs using perpendicularly magnetized metallic magnetic layers using $\mathrm{MgO}$ barrier.

Of late, oxide magnetic materials are emerging as potential candidates for substituting the established metallic spintronics devices because of their versatility and flexibility in tuning the properties which are essential for fabricating superior devices ${ }^{16-18}$. Hence, it is perceived that oxide spintronics may play a vital role in the next generation electronics. In this regard, spin injection into an oxide and from an oxide need to be realized as it is crucial for the development of oxide spintronics. Recently, Wei Han et al.${ }^{19}$ showed that it is possible to inject spins from metallic CoFe into oxide materials such as $\mathrm{La}$ doped or $\mathrm{Nb}$ doped $\mathrm{SrTiO}_{3}$. Now what remains to be addressed is spin-injection from an oxide magnetic material. In this regard, room temperature magnetic oxides like $\mathrm{Fe}_{3} \mathrm{O}_{4}$ and LSMO $\left(\mathrm{La}_{1-\mathrm{x}} \mathrm{Sr}_{\mathrm{x}} \mathrm{MnO}_{3}\right)$ would be the first choice, since they have the advantage of theoretically predicted half metallic nature which makes them perfect candidates for efficient spin injection due to high spin polarization. Stable room temperature ferrimagnetism and high ferrimagnetic Curie temperature $(\sim 860 \mathrm{~K})$ of $\mathrm{Fe}_{3} \mathrm{O}_{4}$ make it more attractive from application point of view in addition to the half metallicity. Moreover, spinpolarized photoelectron spectroscopy experiment on epitaxial $\mathrm{Fe}_{3} \mathrm{O}_{4}$ thin films supports the theoretical prediction of half-metallicity with measured spin polarization $\sim 80 \pm 5 \%{ }^{20}$. In the present study we benchmark the spin injection from $\mathrm{Fe}_{3} \mathrm{O}_{4}$ into the well studied conventional semiconductor $\mathrm{GaAs}$ using $\mathrm{MgO}$ as 
tunnel barrier. In GaAs, the spin relaxation studies have been carried out by various groups in the past from the optical as well as electrical spin injection point of view. Due to the non-centro symmetric crystal structure of GaAs, an additional field in the lattice similar to magnetic field of spin-orbit interaction influences the spin scattering (D'yakonov-Perel mechanism ${ }^{21}$ ) more apart from the usual momentum scattering and impurity scattering when compared with centro symmetric crystals like Si. From earlier reports it is seen that all electrical spin injection and detection using in-plane magnetized ferromagnetic systems in GaAs is observed only up to $\sim 120 \mathrm{~K}^{2-4,22,23}$. In spite of these facts, we have demonstrated spin injection and detection in in-plane magnetized $\mathrm{Fe}_{3} \mathrm{O}_{4} / \mathrm{MgO} / \mathrm{n}$-type GaAs and p-type $\mathrm{GaAs}$ devices at room temperature. In the recent past, T. Saito et. al. ${ }^{24}$ and J. Bae et. al. ${ }^{15}$ were also successful in observing spin signal in GaAs at room temperature by electrically injection method with the help of highly spin polarized material like Heusler alloy and perpendicularly magnetized layer in combination with $\mathrm{MgO}$ barrier respectively. Since we have used an oxide magnet for the demonstration of spin injection, our efforts with spin injection and detection using an oxide material will be of importance in establishing these oxides for the future spintronics devices.

In the recent years, electrical 3-terminal Hanle technique (as depicted in Figure 1) has attracted a lot of attention since it is one of the simple yet powerful techniques to detect spins in semiconductors. Figure 1(a) represents the schematics of the 3-terminal measurement geometry with the device at the centre being common for one of the current as well as voltage lead. Unlike the four terminal measurements, this technique directly probes the spin injection right at the $\mathrm{Fe}_{3} \mathrm{O}_{4} / \mathrm{MgO} / \mathrm{GaAs}$ interface ${ }^{5}$. A constant injection current will help in accumulating these injected spins at the interface between the semiconductor and the barrier. The magnitude of these accumulated spins is given as $\Delta \mu=\mu_{\uparrow}-\mu_{\downarrow}$, where $\mu_{\uparrow}$ and $\mu_{\downarrow}$ are the chemical potentials corresponding to the majority and minority carriers respectively. These accumulated spins precess within the semiconductor as a function of perpendicular magnetic field leading to the decay of the accumulated spins (shown in Figure 1b) with its functional form as a Lorentzian, $\Delta \mu\left(\mathrm{B}_{\mathrm{z}}\right)=\Delta \mu(0) / 1+\left(\omega_{\mathrm{L}} \tau\right)^{2}$, where $\omega_{\mathrm{L}}$ is Larmor frequency of precession of spins, it is defined as $\omega_{L}=g \mu_{B} B_{z} / \hbar$, with Lande's g-factor value of GaAs to be -0.44 and $\tau$ being the effective spin relaxation time. The magnitude of the accumulated spin is measured as $\Delta \mathrm{V}$, which is related to $\Delta \mu$ as $\Delta V=T S P \times \Delta \mu / 2$, where TSP is the tunnel spin polarization of the $\mathrm{Fe}_{3} \mathrm{O}_{4} / \mathrm{MgO}$ combination.

\section{Results}

$\mathrm{Fe}_{3} \mathrm{O}_{4}\left(a_{\mathrm{Fe} 3 \mathrm{O} 4}=8.396 \mathrm{~A}\right)$ also has an interesting low temperature transition at $120 \mathrm{~K}$ known as the Verwey transition ${ }^{25}$, which is a first order transition with structural, magnetic, electrical ordering within the material ${ }^{26}$. Growth of $\mathrm{Fe}_{3} \mathrm{O}_{4}$ and $\mathrm{MgO}$ on $\mathrm{GaAs}$ system follows a perfect epitaxial relation $\mathrm{Fe}_{3} \mathrm{O}_{4}$ (100) [011]//MgO (100) [001]//GaAs (100) $[001]^{27,28}$ in addition to all the attractive features listed above. With $a_{\mathrm{MgO}}=4.212 \mathrm{~A}$, the lattice mismatch between $\mathrm{Fe}_{3} \mathrm{O}_{4}$ and $\mathrm{MgO}$ is only about only $0.33 \%$ because of the $45^{\circ}$ in-plane rotation growth of $\mathrm{Fe}_{3} \mathrm{O}_{4}$. In our study, a stack of $\mathrm{Fe}_{3} \mathrm{O}_{4}$ and $\mathrm{MgO}$ thin films were grown on $\mathrm{Si}$ - doped n-type GaAs with $1 \times 10^{18} / \mathrm{cm}^{3}$ doping and $\mathrm{Zn}$ doped p-type GaAs with $1 \times 10^{19} / \mathrm{cm}^{3}$ (see the methods for sample preparation). SEM micrograph of the typical Hanle structure is as shown in Figure 1 (c). The quality of $\mathrm{Fe}_{3} \mathrm{O}_{4} / \mathrm{MgO} / \mathrm{GaAs}$ interface has been verified with TEM, and the typical image is shown in Figure 1(d). From the X-ray diffraction measurements, it is seen that the growth of $\mathrm{Fe}_{3} \mathrm{O}_{4} / \mathrm{MgO}$ on GaAs is oriented (Figure $\mathrm{S} 1$ of supplementary material) and epitaxial. Figure $\mathrm{S} 2$ of supplementary material further confirms the $\mathrm{Fe}_{3} \mathrm{O}_{4}$ film growth and the absence of satellite peak at $719 \mathrm{eV}$ which confirms the absence of $\mathrm{Fe}_{2} \mathrm{O}_{3}$. Also, the mag-

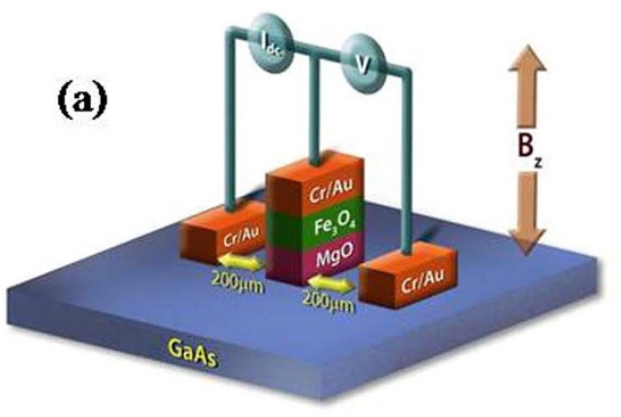

(c)

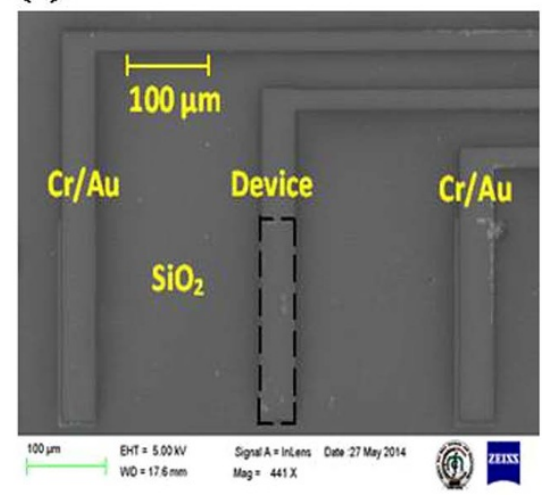

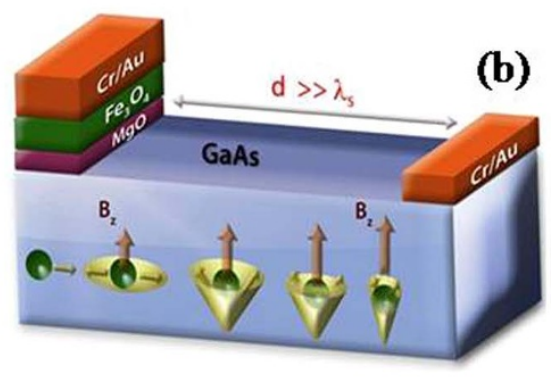

(d)

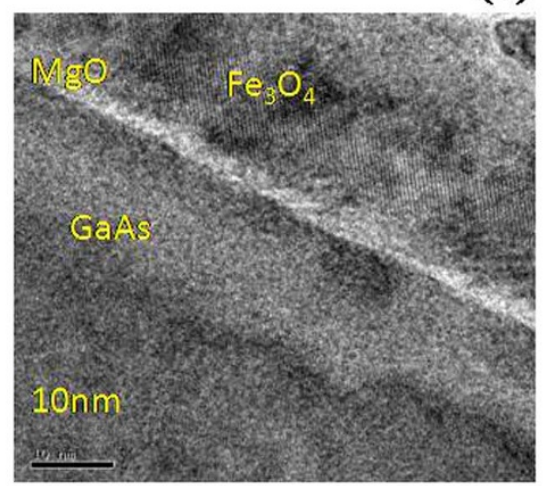

Figure 1 Schematics of 3-terminal Hanle measurement geometry. (a) The structured devices of dimension $50 \times 200 \mu \mathrm{m}^{2}$ are separated by $\mathrm{d}=200 \mu \mathrm{m}$ distance between the contact pads with $\mathrm{d} \gg \lambda_{s}$. (b) With constant DC current $\mathrm{I}_{\mathrm{dc}}$ flowing, accumulated $\Delta \mu$ precesses about $\mathrm{B}_{\mathrm{z}}$. As the strength of the applied field increases, the accumulated spins get dephased and relax close to the direction of the applied magnetic field. (c) Typical SEM image of the Hanle structure. (d) TEM micrograph of the $\mathrm{Fe}_{3} \mathrm{O}_{4} / \mathrm{MgO} / \mathrm{GaAs}$ film. 
netization as a function of temperature (Figure S3(a) of supplemental material) of the film shows a very sharp Verwey transition and also the $\log \mathrm{R}$ vs. $\mathrm{T}$ plot as shown in Figure $\mathrm{S} 4$ confirms the superior quality of $\mathrm{Fe}_{3} \mathrm{O}_{4}$ film.

Spin injection into $\mathrm{n}-\mathrm{GaAs}$ using $\mathrm{Fe}_{3} \mathrm{O}_{4}$. Investigation of spin injection and accumulation into $\mathrm{n}$-GaAs using $\mathrm{Fe}_{3} \mathrm{O}_{4} / \mathrm{MgO}$ injection system is discussed in detail at first. Figure 2 shows the transport measurements carried out on the photlithographically structured $50 \times 200 \mu \mathrm{m}^{2}$ devices of $\mathrm{Fe}_{3} \mathrm{O}_{4} / \mathrm{MgO} / \mathrm{n}$-type $\mathrm{GaAs}$ and the Hanle measurements results are plotted in Figure 2(a), (b) and (c). We have observed $\Delta \mathrm{V}$ of $\sim 0.35 \mathrm{mV}$ for an injection current of $1 \mu \mathrm{A}$. From Figure 2(a), it is seen that the accumulated spins decay as the strength of $B_{z}$ increases. Lorentzian fit to the curve yields a $\tau$ value of $\sim 0.9 \mathrm{~ns}$. This is a lower bound value of the spin relaxation time due to the broadening of the Hanle curve, as discussed by Dash S. P. et $a^{22}$. At higher fields, the magnitude of $\Delta \mathrm{V}$ increases again because of the rotation of the magnetization axis to out of plane for $\mathrm{Fe}_{3} \mathrm{O}_{4}$ layer. The spin resistance $\Delta \mathrm{R}$ for the corresponding $\Delta \mathrm{V}$ is calculated as $\Delta \mathrm{R}=\Delta \mathrm{V} / \mathrm{I}$. The spin resistance-area product $\Delta R \times A$ in $\mathrm{k} \Omega \mu \mathrm{m}^{2}$ vs. $\mathrm{B}_{\mathrm{z}}$ for different injection currents are plotted in Figure $2(\mathrm{~b})$ so that the magnitude of spin signals can be compared clearly. The FWHM of the curves at different injection currents remain same which clearly indicate the genuineness of the $\tau$ value extracted. Hanle measurements were repeated at $80 \mathrm{~K}$ and the data is shown in Figure 2(c). It is seen that the $\tau$ value has increased to $\sim 1.6 \mathrm{~ns}$ with a huge $\Delta \mathrm{V}$ of $\sim 18 \mathrm{mV}$. A systematic study of $\tau$ extracted as a function of temperature is plotted in Figure 2(d). Since the resistivity of $\mathrm{Fe}_{3} \mathrm{O}_{4}$ becomes very high at lower temperatures $(\mathrm{T}<70 \mathrm{~K})$, measurements are carried out at temperatures $\mathrm{T} \geq 80 \mathrm{~K}$. As expected, the value of $\tau$ decays from $1.6 \mathrm{~ns}$ to $0.9 \mathrm{~ns}$ from $80 \mathrm{~K}$ to $300 \mathrm{~K}$ due to the increased lattice scattering.
Spin injection into p-GaAs using $\mathrm{Fe}_{3} \mathrm{O}_{4}$. Figure 3(a) shows the plot of $\Delta \mathrm{V}$ vs. $\mathrm{B}_{\mathrm{z}}$ of $\mathrm{Fe}_{3} \mathrm{O}_{4} / \mathrm{MgO} / \mathrm{p}-\mathrm{GaAs}$ device of area $50 \times 200 \mu \mathrm{m}^{2}$ at $300 \mathrm{~K}$. I-V measurements on the sample at two different temperatures are plotted in Figure S5 of supplementary material. We have observed the accumulation of spins even in $\mathrm{p}-\mathrm{GaAs}$, and this is evident from a $\Delta \mathrm{V}$ as high as $5 \mu \mathrm{V}$ for $5 \mu \mathrm{A}$ extraction current at room temperature in GaAs as shown in the inset of Figure 3(a). It can be seen that though the accumulation of spin are about 2 order lower than that of $n$-type, still a detectable injection of spins and the decay of spin is observed. Figure $3(b)$ is the plot of $\Delta R$ vs. $B_{z}$ for different extraction currents. As $B_{z}$ increases, dephasing results in the decay of the accumulated spins under the $\mathrm{Fe}_{3} \mathrm{O}_{4} / \mathrm{MgO}$ layer. The value of the hole spin relaxation time from the Lorentzian fit at room temperature is found to be $\sim 0.32 \mathrm{~ns}$; (assuming that the $\mathrm{g}$ value for holes to be 0.44 , same as for electrons). Also the measurements at $80 \mathrm{~K}$ yield a systematic variation of the Hanle data for the junction. A spin relaxation time of $\sim 0.6$ ns was obtained from the Lorentzian equation mentioned above which is almost double of the value of $\tau$ at room temperature. In Figure 3(d), the $\Delta R \times A$ product of the junction at 2 different temperatures is compared. As the temperature is increased from $80 \mathrm{~K}$ to room temperature, there is a clear diminution in spin relaxation time to about $0.6 \mathrm{~ns}$ from $0.32 \mathrm{~ns}$. It is quite understandable that the spins get relaxed more because of the increased lattice scattering due to temperature factor. A plot of the relaxation time as a function of extraction current at 300 and $80 \mathrm{~K}$ is given in the Figure S6 of the supplementary material.

It has to be noted that the obtained value of $\Delta \mathrm{V}$ is weak for $\mathrm{p}-\mathrm{GaAs}$ when compared with $\mathrm{n}$-GaAs. Hence, in order to check the authenticity of the values obtained, we have done further measurements on different samples by varying the $\mathrm{MgO}$ barrier thickness. Figure 4 shows Hanle data for $\mathrm{Fe}_{3} \mathrm{O}_{4} / \mathrm{MgO}(\mathrm{x}=1,1.5,3 \mathrm{~nm}) / \mathrm{p}-\mathrm{GaAs}$ at room
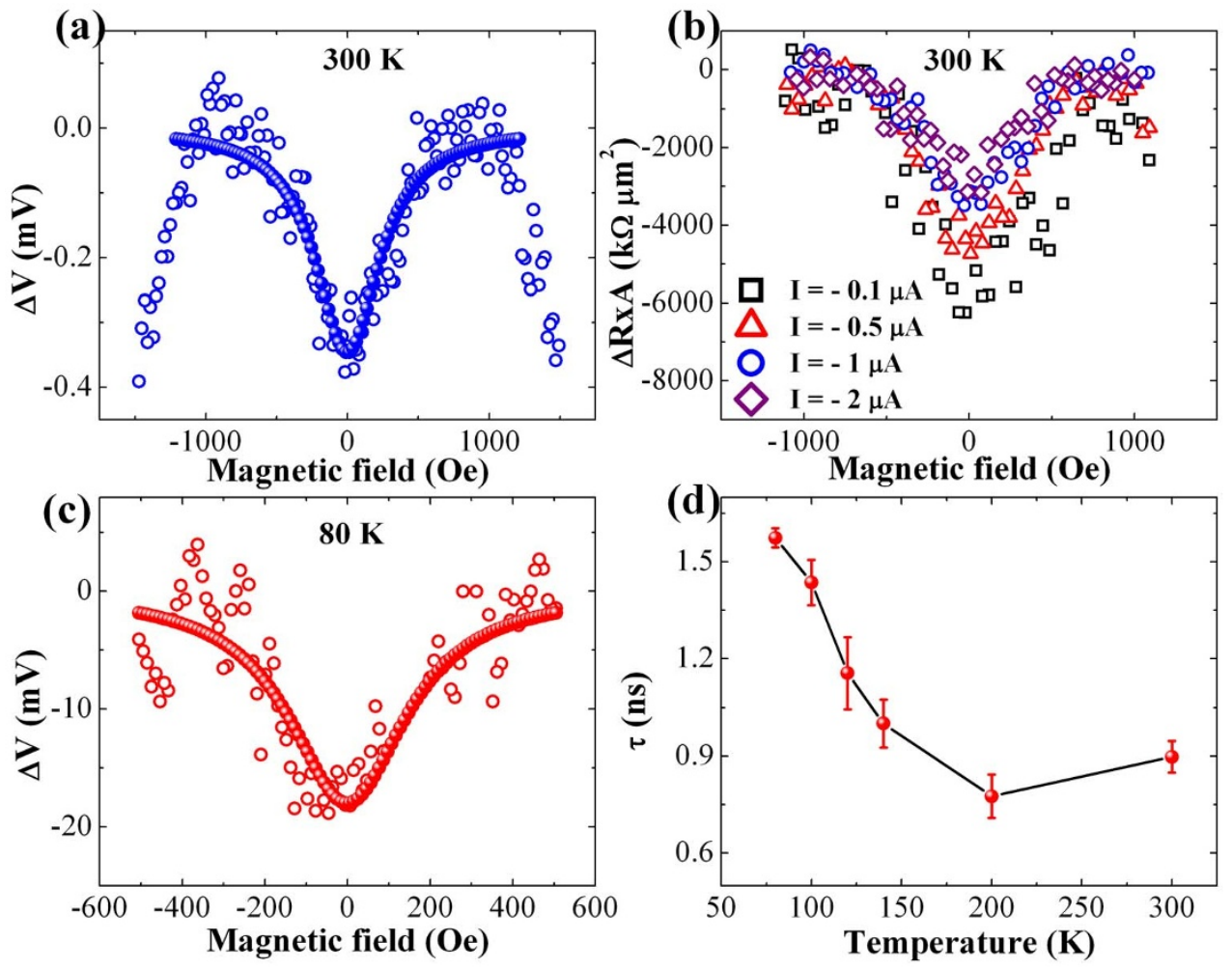

Figure 2 Electrical Hanle measurements on $\mathrm{Fe}_{3} \mathrm{O}_{4} / \mathrm{MgO} / \mathrm{n}$-GaAs. (a) Hanle data of the film (circular open symbols) for $1 \mu \mathrm{A}$ electron spin injection current at $300 \mathrm{~K}$. Plot of $\Delta V$ vs. $\mathrm{B}_{\mathrm{z}}$ shows a spin accumulation of $\sim 0.35 \mathrm{mV}$. (b) $\Delta R \times A\left(\mathrm{k} \Omega \mu \mathrm{m}^{2}\right) \mathrm{vs}$. $\mathrm{B}_{\mathrm{z}}$ for different electron spin injection currents for 50 $\times 200 \mu \mathrm{m}^{2}$ area devices. (c) $\Delta V$ vs. $B_{z}$ plot at $80 \mathrm{~K}$ is observed to be having lesser FWHM in comparison to $300 \mathrm{~K}$ data. (d) Spin relaxation time $\tau$ of $\mathrm{n}-\mathrm{GaAs}$ as a function of temperature (circular solid symbols). The line drawn is just guide to eye. 

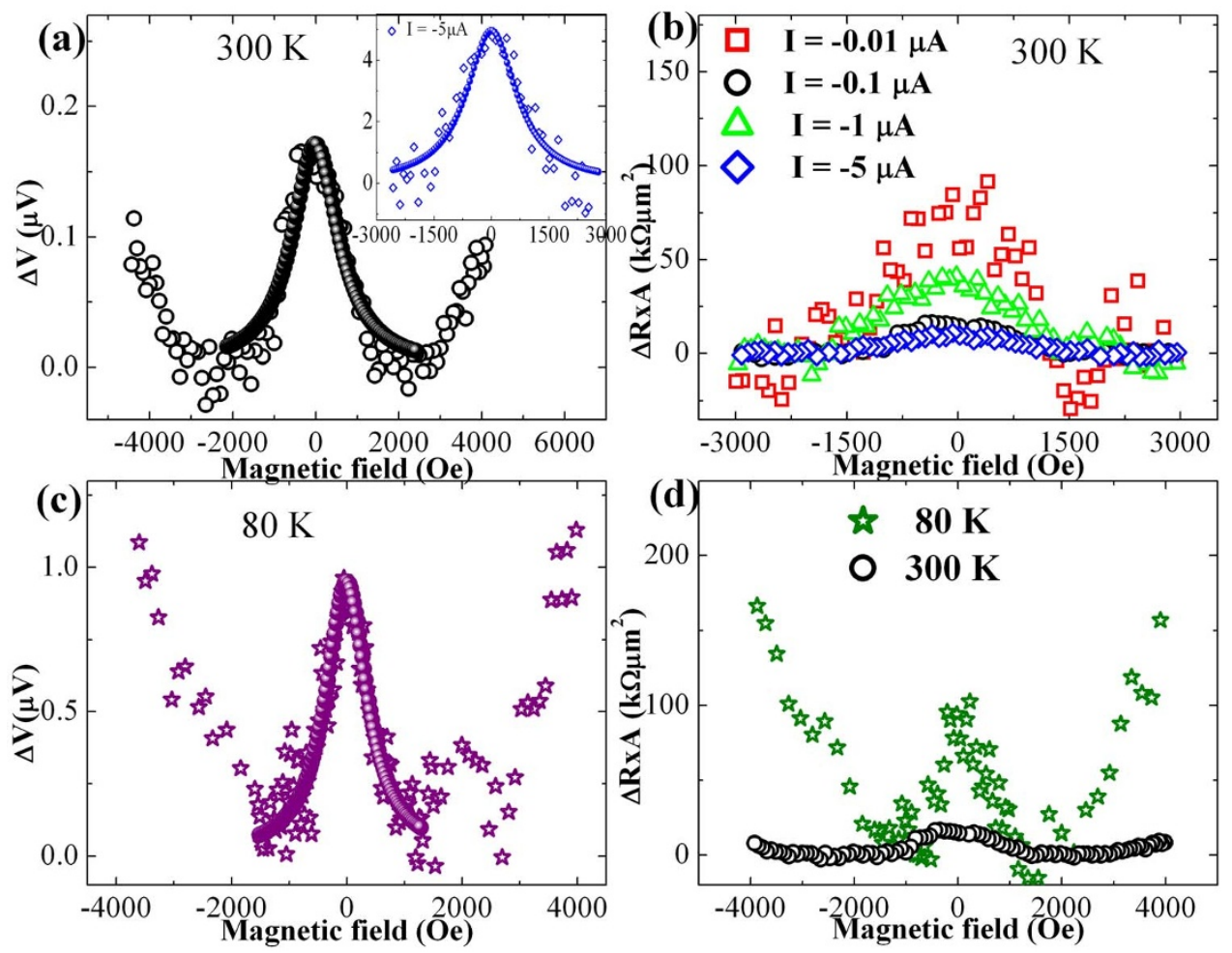

Figure 3 Electrical Hanle measurement on $\mathrm{Fe}_{3} \mathrm{O}_{4} / \mathrm{MgO} / \mathrm{p}$-GaAs devices. (a) Plot of $\Delta \mathrm{V}$ vs. $\mathrm{B}_{\mathrm{z}}$ at $300 \mathrm{~K}$ at $0.1 \mu \mathrm{A}$ (circular open symbols) with Hanle fit (circular solid symbols). Inset shows a maximum spin accumulation of $5 \mu \mathrm{V}$ for an electron injection current of $5 \mu \mathrm{A}$ (diamond open symbols). (b) Plot of $\Delta R \times A$ product $\left(\mathrm{k} \Omega \mu \mathrm{m}^{2}\right)$ vs. $\mathrm{B}_{\mathrm{z}}$ at $300 \mathrm{~K}$ at various hole extraction currents for the devices with $50 \times 200 \mu \mathrm{m}^{2}$ area. (d) Plot of $\Delta \mathrm{V}$ vs. $\mathrm{B}_{\mathrm{z}}$ at $80 \mathrm{~K}$ at $0.01 \mu \mathrm{A}$ (star open symbols). (e) Comparison of $\Delta R \times A$ product $\left(\mathrm{k} \Omega \mu \mathrm{m}^{2}\right)$ at 80 and $300 \mathrm{~K}$ (star open symbols and circular open symbols respectively) at extraction current of $0.1 \mu \mathrm{A}$ with different FWHM for the curves.

temperature. It is seen from the Figure 4 that the FWHM for different thickness of $\mathrm{MgO}$ remains almost similar indicating that there is no change in $\tau$ values extracted individually for these different samples. In all the cases, the average value of $\tau$ is $\sim 0.29 \pm 0.03 \mathrm{~ns}$. Hence, the spin relaxation time obtained for $\mathrm{p}-\mathrm{GaAs}$ is free of any extrinsic effects of the junction.

\section{Discussion}

From the demonstration of the spin injection in GaAs at room temperature as well as at low temperatures, we can see that the $\mathrm{Fe}_{3} \mathrm{O}_{4}$ / $\mathrm{MgO}$ oxide system is highly efficient for the spin injection studies. We obtain a $\Delta \mathrm{V}$ of $\sim 0.3 \mathrm{mV}$ at $300 \mathrm{~K}$ which is in fact comparable to $\Delta \mathrm{V}$ obtained using $3 \mathrm{TH}$ measurements in $\mathrm{Si}^{5,6,29}$ than $\Delta \mathrm{V}$ in $\mathrm{GaAs}^{15,30,31}$ and at low temperatures, $\Delta \mathrm{V}$ as high as $\sim 18 \mathrm{mV}$ is observed. Apart from having a high spin polarization from $\mathrm{Fe}_{3} \mathrm{O}_{4}$, the spin injection and detection at room temperature as well as at low temperatures in GaAs might be attributed to the coherent spin tunneling due to the presence of symmetry states in $\mathrm{MgO}^{32}$. The $\Delta$ symmetry bands of $\mathrm{MgO}$ is known to be preserving the spins from scattering within $\mathrm{MgO}$, and we believe that this could be the prime factor for observing a spin signal of $\sim \mathrm{mVs}$ in GaAs in spite of its strong spin scattering near room temperature. One of the recent reports also support this fact where in similar scenario of $\mathrm{MgO}$ is discussed with the perpendicularly magnetized metallic layer ${ }^{15}$. This shows that the presence of $\mathrm{MgO}$ in this spin injection system is necessary to inject as well as detect the spins in GaAs at room temperature. In $\mathrm{p}-\mathrm{GaAs}$ it is seen that the magnitude of $\Delta \mathrm{V}$ observed increases from $\sim 0.15 \mu \mathrm{V}$ to $\sim 0.8 \mathrm{mV}$ with the barrier thickness. Hence it is clear that the increase in junction resistance improves the value of accumulated spin. Increase in the symmetry filtering effect introduced by $\mathrm{MgO}$ for increased thickness of $\mathrm{MgO}$ cannot be fully ruled out in this context.
In summary, we demonstrate the spin injection into the conventional semiconductor $\mathrm{GaAs}$ using an oxide magnetic material, $\mathrm{Fe}_{3} \mathrm{O}_{4}$, with the help of a tunnel barrier. The spin relaxation time for $\mathrm{n}-\mathrm{GaAs}$ is found to be $\sim 0.9 \mathrm{~ns}$ at room temperature. The temperature

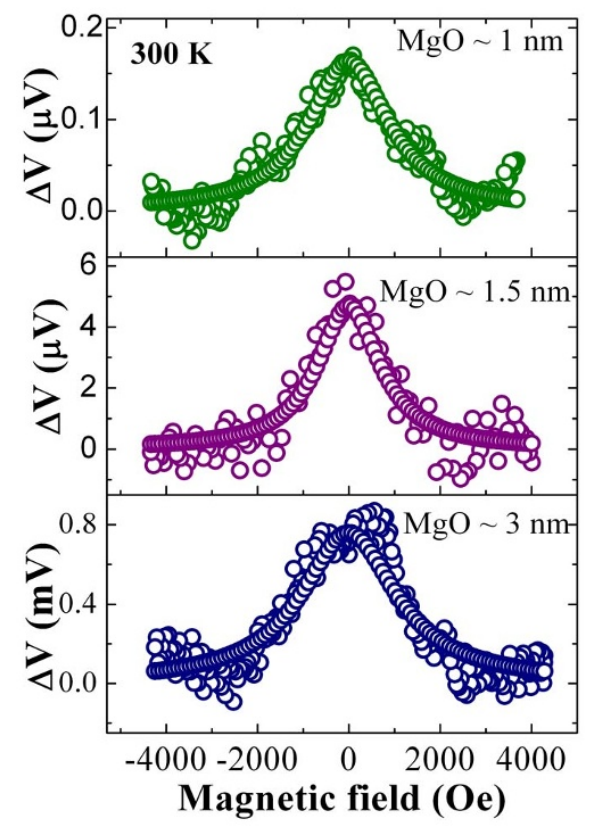

Figure $4 \mid$ Electrical Hanle measurement on $\mathrm{Fe}_{3} \mathrm{O}_{4} / \mathrm{MgO}(\mathrm{x}=1,1.5$, $3 \mathrm{~nm}) / \mathrm{p}$-GaAs devices. With different MgO barrier thickness, the FWHM of the curves obtained for different devices are of the same order of extracted spin relaxation time. The value of $\tau$ averages out to be $0.29 \pm$ 0.03 ns. 
dependent study of Hanle signal indicates the increment in $\tau$ value for decreasing the temperature as expected. Also, the demonstration of spin injection in $\mathrm{p}-\mathrm{GaAs}$ strengthens the fact of high injection efficiency of $\mathrm{Fe}_{3} \mathrm{O}_{4} / \mathrm{MgO}$ combination. A spin relaxation time of $\sim 0.32 \mathrm{~ns}$ is obtained for $\mathrm{p}-\mathrm{GaAs}$ at room temperature, whereas there is a clear enhancement in the $\tau$ value to $\sim 0.6 \mathrm{~ns}$ at $80 \mathrm{~K}$. Hanle measurements for different thickness of $\mathrm{MgO}$ barrier on $\mathrm{p}$-GaAs at room temperature also indicates the same relaxation time of $\sim 0.29$ $\pm 0.03 \mathrm{~ns}$, ruling out the extrinsic effect of observing the signal in $\mathrm{p}$ GaAs. All these experiments establish $\mathrm{Fe}_{3} \mathrm{O}_{4} / \mathrm{MgO}$ combination as a very good spin injection system. We thus conclude that even oxide magnetic materials are also equally attractive, promising, and capable of establishing a field of spin injection and detection in the area of oxides.

\section{Methods}

Sample preparation. Pulsed laser ablation technique is used to deposit the film stack of $\mathrm{Fe}_{3} \mathrm{O}_{4} / \mathrm{MgO}$ on GaAs. Substrate is $\mathrm{Zn}$ - doped p-type GaAs with a carrier concentration specified from the manufacturer to be $1 \times 10^{19} / \mathrm{cm}^{3}$. Prior to the deposition, substrate was cleaned with acetone and IPA. Substrate was subsequently dipped in $1 \% \mathrm{HF}$ in order to remove the native oxide on GaAs. Then the substrate was loaded into the deposition chamber and annealed at $500^{\circ} \mathrm{C}$ to get uniform and clean surface of GaAs. Different thicknesses of $\mathrm{MgO}$ (for different samples) were deposited at $500^{\circ} \mathrm{C}$ followed by a deposition of $30 \mathrm{~nm}$ of $\mathrm{Fe}_{3} 0_{4}$ at $450^{\circ} \mathrm{C}$. The post annealing of the film was carried out at $450^{\circ} \mathrm{C}$ for an hour in vacuum of the order $10^{-5} \mathrm{~m}$ bar.

Sample fabrication. In order to get 3-terminal Hanle geometry for the transport measurement, these continuous films were structured using photolithography followed by Ar ion beam etching. About $100 \mathrm{~nm}$ of $\mathrm{SiO}_{2}$ layer was deposited for the isolation of the defined device area. Then a $2^{\text {nd }}$ level of photolithography and deposition were carried out to define the contact pad, $\mathrm{Cr}(10 \mathrm{~nm}) / \mathrm{Au}(100 \mathrm{~nm})$. Alwedge bonding was used to connect the electrical leads to these devices.

1. Datta, S. \& Das, B. Electronic analog of the electro-optic modulator. Appl. Phys Lett. 56, 665 (1990).

2. Lou, X. et al. Electrical detection of spin accumulation at a ferromagnetsemiconductor interface. Phys. Rev. Lett. 96, 176603 (2006)

3. Lou, X. et al. Electrical detection of spin transport in lateral ferromagnetsemiconductor devices. Nature Phys. 3, 197-202 (2007)

4. Tran, M. et al. Enhancement of the spin accumulation at the interface between a spin-polarized tunnel junction and a semiconductor. Phys. Rev. Lett. 102, 036601 (2009).

5. Dash, S. P., Sharma, S., Patel, R. S., Jong, M. P. \& Jansen, R. Electrical creation of spin polarization in silicon at room temperature. Nature 462, 491-494 (2009).

6. Li, C. H., van't Erve, O. M. J. \& Jonker, B. T. Electrical injection and detection of spin accumulation in silicon at $500 \mathrm{~K}$ with magnetic metal/silicon oxide contacts. Nat. Commun. 2, 245 (2011).

7. Fiederling, R. et al. Injection and detection of a spin-polarized current in a lightemitting diode. Nature 402, 787-790 (1999).

8. Zhu, H. J. et al. Room-Temperature Spin Injection from Fe into GaAs. Phys. Rev. Lett. 87, 016601 (2001)

9. Ohno, Y. et al. Electrical spin injection in a ferromagnetic semiconductor heterostructure. Nature 402, 790-792 (1999).

10. Hanbicki, A. T., Jonker, B. T., Itskos, G., Kioseoglou, G. \& Petrou, A. Efficient electrical spin injection from a magnetic metal/tunnel barrier contct into a semiconductor. Appl. Phys. Lett. 80, 1240 (2002).

11. Jiang, X. et al. Highly spin-polarized room-temperature tunnel injector for semiconductor spintronics using $\mathrm{MgO}$ (100). Phys. Rev. Lett. 94, 056601 (2005).

12. Jedema, F. J., Heersche, H. B., Filip, A. T., Baselmans, J. J. A. \& van Wees, B. J. Electrical detection of spin precession in a metallic mesoscopic spin valve. Nature 416, 713 (2002).

13. Rashba, E. I. Theory of electrical spin injection: Tunnel contacts as a solution of the conductivity mismatch problem. Phys. Rev. B. 62, R16267-R16270 (2000).

14. Fert, A. \& Jaffres, H. Conditions for efficient spin injection from a ferromagnetic metal into a semiconductor. Phys. Rev. B 64, 184420 (2001).

15. Bae, J. et al. Transport of perpendicular spin in a semiconductor channel via a fully electrical method. Appl. Phy. Lett. 102, 062412 (2013).
16. Tian, Y., Bakaul, S. R. \& Wu, T. Oxide nanowires for spintronics: materials and devices. Nanoscale. 4, 1529-1540 (2012).

17. Reyren, N. et al. Gate-Controlled Spin Injection at $\mathrm{LaAlO}_{3} / \mathrm{SrTiO}_{3}$ Interfaces. Phys. Rev. Lett. 108, 186802 (2012).

18. Pullizi, F. Oxidized spintronics. Nat. Mater. 11, 564 (2012).

19. Han, W. et al. Spin injection and detection in lanthanum- and niobium-doped $\mathrm{SrTiO}_{3}$ using Hanle technique. Nat. Commun. 4, 2134 (2013).

20. Dedkov, Y. S., Rüdiger, U. \& Güntherodt, G. Evidence for the half-metallic ferromagnetic state of $\mathrm{Fe}_{3} \mathrm{O}_{4}$ by spin-resolved photoelectron spectroscopy. Phys. Rev. B. 65, 064417 (2002)

21. Dyakonov, M. I. \& Perel, V. I. Spin relaxation of conduction electrons in noncentrosymmetric semiconductors. Sov.Phys. Solid State. 13, 3023 (1972).

22. Dash, S. P. et al. Spin precession and inverted Hanle effect in semiconductor near a finite-roughness ferromagnetic interface. Phys. Rev. B. 84, 054410 (2011).

23. Fleet, L. R. et al. Correlating the interface structure to spin injection in abrupt $\mathrm{Fe} /$ GaAs (001) films. Phys. Rev. B. 87, 024401 (2013).

24. Saito, T., Tezuka, N., Matsuura, M. \& Sugimoto, S. Spin injection, transport, and detection at room temperature in a lateral spin transport device with $\mathrm{Co}_{2} \mathrm{FeAl}_{0.5} \mathrm{Si}_{0.5} / \mathrm{n}-\mathrm{GaAs}$ schottky tunnel junctions. Appl. Phys. Express. 6, 103006 (2013).

25. Verwey, E. J. W. Electronic conduction of magnetite $\left(\mathrm{Fe}_{3} \mathrm{O}_{4}\right)$ and its transition point at low temperatures. Nature 144, 327-328 (1939).

26. Walz, F. The Verwey transition-a topical review. J. Phys.: Condens. Matter. 14, R285-R340 (2002).

27. Wong, P. K. J., Zhang, W., Xu, Y., Hassan, S. \& Thompson, S. M. Magnetic and structural properties of fully epitaxial $\mathrm{Fe}_{3} \mathrm{O}_{4} / \mathrm{MgO} / \mathrm{GaAs}(100)$ for spin injection. IEEE Trans. on Magn. 44, 2640-2642 (2008).

28. Lu, Y.X. et al. Epitaxial growth and magnetic properties of half-metallic $\mathrm{Fe}_{3} \mathrm{O}_{4}$ on GaAs (100). Phys. Rev. B. 70, 233304 (2004).

29. Jeon, K.-R. et al. Electrical spin accumulation with improved bias voltage dependence in a crystalline $\mathrm{CoFe} / \mathrm{MgO} / \mathrm{Si}$ system. Appl. Phy. Lett. 98, 262102 (2011).

30. Bruski, P. et al. All-electrical spin injection and detection in the $\mathrm{Co}_{2} \mathrm{FeSi} / \mathrm{GaAs}$ hybrid system in the local and non-local configuration. Appl. Phy. Lett. 103, 052406 (2013).

31. Saito, T., Tezuka, N. \& Sugimoto, S. Electrical transport properties and spin injection in $\mathrm{Co}_{2} \mathrm{FeAl}_{0.5} \mathrm{Si}_{0.5} / \mathrm{GaAs}$ junctions. IEEE Trans. on Magn. 47, 2447-2450 (2011).

32. Butler, W. H., Zhang, X.-G. \& Schulthess, T. C. Spin-dependent tunneling conductance of $\mathrm{Fe}|\mathrm{MgO}| \mathrm{Fe}$ sandwiches. Phys. Rev. B 63, 054416 (2001).

\section{Acknowledgments}

This work is financially supported by DST, INDIA. We acknowledge NNfC, CeNSE, IISc for the nanofabrication facility. One of the authors S.G.B. extends her gratitude to CSIR, INDIA for the financial support.

\section{Author contributions}

S.G.B. performed the experiments and the subsequent measurements. P.S.A.K. supervised and coordinated the project and contributed towards the insights and ideas of the project. S.G.B. prepared the manuscript with inputs from P.S.A.K.

\section{Additional information}

Supplementary information accompanies this paper at http://www.nature.com/ scientificreports

Competing financial interests: The authors declare no competing financial interests. How to cite this article: Bhat, S.G. \& Kumar, P.S.A. Room temperature electrical spin injection into GaAs by an oxide spin injector. Sci. Rep. 4, 5588; DOI:10.1038/srep05588 (2014).

This work is licensed under a Creative Commons Attribution-NonCommercialNoDerivs 4.0 International License. The images or other third party material in this article are included in the article's Creative Commons license, unless indicated otherwise in the credit line; if the material is not included under the Creative Commons license, users will need to obtain permission from the license holder in order to reproduce the material. To view a copy of this license, visit http:// creativecommons.org/licenses/by-nc-nd/4.0/ 
SUBJECT AREAS: SPINTRONICS

ELECTRONIC DEVICES

\section{SCIENTIFIC REPORTS:}

$4: 5588$

DOI: $10.1038 /$ srep05588

(2014)

Published: 7 July 2014

Updated: 14 October 2014
CORRIGENDUM: Room temperature electrical spin injection into GaAs by an oxide spin injector

\author{
Shwetha G. Bhat \& P. S. Anil Kumar
}

The following sentence was inaccurate and has been removed from the abstract: "So far there is no success in using a magnetic oxide material for spin injection, which is very important for the development of oxide based spintronics devices."

The following sentence in the introduction was inaccurate: "From earlier reports it is seen that spin injection using in-plane magnetized ferromagnetic systems in GaAs is observed only up to $\sim 120 \mathrm{~K}^{2-4,22,23}$." It has been changed to: "From earlier reports it is seen that all electrical spin injection and detection using in-plane magnetized ferromagnetic systems in GaAs is observed only up to $\sim 120 \mathrm{~K}^{2-4,22,23}$." 